CARNETS DE Carnets de géographes

GÉOGRAPHES.

5 | 2013

Géographie humanimale

\title{
Le paysage, levier d'action dans la planification territoriale
}

Essai d'évaluation de la politique paysagère du SCOT de l'aire métropolitaine de Bordeaux

Didier Labat

\section{(2) OpenEdition}

Journals

Édition électronique

URL : http://journals.openedition.org/cdg/1105

DOI : $10.4000 / \mathrm{cdg} .1105$

ISSN : 2107-7266

Éditeur

UMR 245 - CESSMA

Référence électronique

Didier Labat, «Le paysage, levier d'action dans la planification territoriale », Carnets de géographes [En

ligne], 5 | 2013, mis en ligne le 01 janvier 2013, consulté le 23 septembre 2020. URL : http://

journals.openedition.org/cdg/1105; DOI : https://doi.org/10.4000/cdg.1105

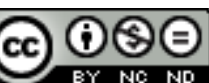

La revue Carnets de géographes est mise à disposition selon les termes de la Licence Creative Commons Attribution - Pas d'Utilisation Commerciale - Pas de Modification 4.0 International. 


\title{
LE PAYSAGE, LEVIER D'ACTION DANS LA PLANIFICATION TERRITORIALE
}

\author{
Essai d'évaluation de la politique paysagère du SCOT de l'aire \\ métropolitaine de Bordeaux
}

\section{DIDIER LABAT}

L'évolution réglementaire des années 1990 à 2000 a positionné le paysage comme élément fondant le cadre de vie dans les outils de planification territoriale. Les acteurs sont incités à les mobiliser dans un contexte territorial concurrentiel et de gouvernance multi-niveaux. Dès lors, se pose la question de l'identification des ressources de ces territoires de projet pour en développer l'attractivité. L'hypothèse principale de cette thèse a été de considérer que le paysage est mobilisé par les acteurs comme un levier d'action publique pour induire des logiques d'aménagement et justifier les fondements du projet de planification. Pour la démonter, nous avons voulu comprendre comment les acteurs d'un instrument de planification s'emparent du paysage pour organiser leur développement territorial sur l'aire métropolitaine de Bordeaux.

C'est à partir de l'analyse de l'action publique et de l'évaluation des politiques publiques que nous avons construit notre corpus méthodologique (schématisé en figure 1). Il nous fallait expérimenter une démarche d'évaluation adaptée à un document d'urbanisme. 
Figure 1 : Synoptique de la démarche de recherche et de l'évaluation engagée.

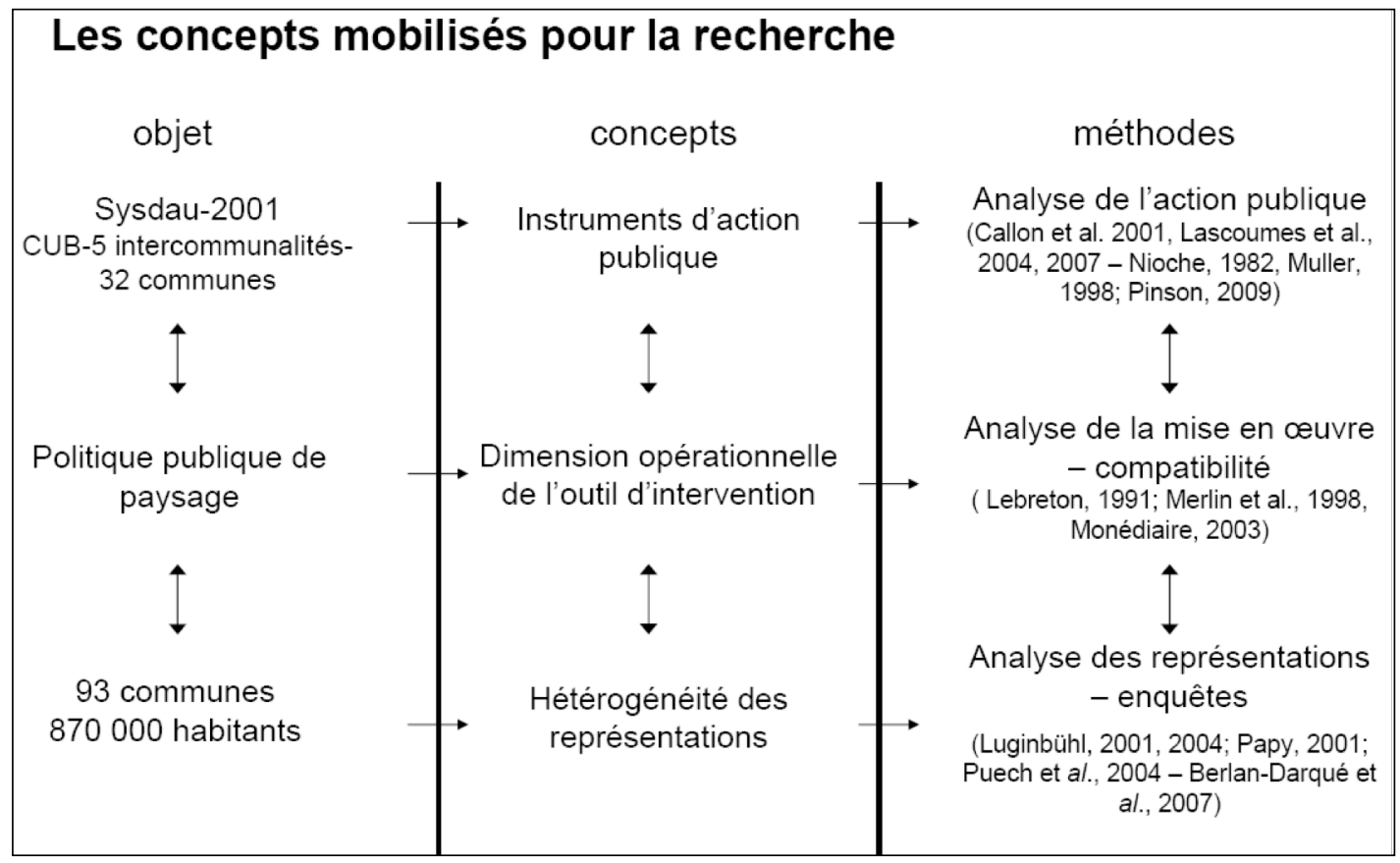

Source : D. Labat - 2011

Nous avons sélectionné le schéma de cohérence territoriale de l'aire métropolitaine de Bordeaux pour expérimenter cette évaluation construite selon quatre étapes :

- une analyse historique des politiques paysagères mises en œuvre dans le cadre de la planification métropolitaine. Depuis les années 1960, les acteurs de cette planification ont fait sensiblement évoluer leur démarche paysagère d'une politique des espaces verts à une politique paysagère intégrée dans le projet de territoire. Le paysage est investi par les collectivités territoriales à partir de l'atlas des paysages en 1997. Il entraîne une évolution significative des représentations du territoire de projet. Une nouvelle recherche d'équilibre entre préservation des paysages et développement métropolitain est construite. La structure du projet fait appel à la formulation d'un véritable projet de paysage dans lequel des objectifs de qualité paysagère sont définis et illustrés dans un niveau de détail inhabituel pour des outils stratégiques de ce type.

\section{- la reconstitution des théories de l'action pour identifier les types de mobilisation des} acteurs (méthodes, réseaux, formulations). Le paysage sert de levier pour introduire les enjeux de l'économie viticole et un discours axé sur le marketing territorial. Le document contient des concepts paysagers innovants favorisant l'articulation entre les politiques urbaines et rurales. Il sert de médiation pour sensibiliser l'ensemble des acteurs sur la maîtrise de l'étalement urbain et sur les rapports urbain/périurbain, sur les franges agricoles/naturelles/forestières de la métropole. Le paysage devient une ressource pour la révélation d'une identité métropolitaine. 
- l'étude de la compatibilité des orientations entre les échelles d'action métropolitaine et communale. Trois plans locaux d'urbanisme ont été analysés pour identifier les correspondances et les ruptures entre les échelles métropolitaines et locales. La comparaison entre les échelles et les outils d'action montre plusieurs éléments qui participent d'une réelle difficulté de mise en œuvre des objectifs métropolitains à l'échelle communale. Les concepts paysagers métropolitains ne sont que partiellement repris dans le PLU. Parfois, leur traduction fait l'objet d'une simplification qui en édulcore le sens initial, voire le détourne à des fins contraires. Les documents locaux sont en réalité construits selon une forte indépendance visà-vis du SD 2001 en ce qui concerne le paysage. Si les limites des enveloppes urbanisables sont respectées, l'élaboration des orientations paysagères est trop étroitement liée à la motivation des élus locaux d'un urbanisme pragmatique et répondant aux enjeux sociaux locaux.

- l'analyse des représentations via deux enquêtes aux deux échelles d'action pour identifier les points de blocage et la cohérence entre les orientations paysagères et les attentes des échantillons. Le regard porté sur les dynamiques urbaines est parfois catégorique quant à la capacité des élus de maîtriser les impacts les plus négatifs. Les idées de projet sont nombreuses et mettent en scène un paysage à la fois protégé dans sa configuration «naturelle » selon les représentations, et à la fois vivant, dans sa configuration sociale lorsqu'il s'agit des espaces publics et habités. Ces représentations traduisent le souhait d'une structure urbaine qui favorise les rencontres, le lien social. Cependant, leur expression témoigne également d'un discours qui se trouve mobilisé à des fins politiques et sociales (concurrence électorale, contestation idéologique, conflits interpersonnels, effet nimby, etc.) et des fins économiques (rentes foncières). Les contradictions identifiées dans le discours de ces personnes montrent le besoin d'une participation qui s'appuie sur des démarches pédagogiques entre expertises techniques et profanes.

Notre expérimentation participe d'un approfondissement des connaissances sur l'évaluation des documents d'urbanisme et des politiques paysagères non contractuelles dans un contexte grandissant d'une demande évaluative des politiques d'aménagement du territoire. Nous avons également mobilisé l'analyse des représentations comme matériau d'évaluation dans un objectif de définition de la pertinence du projet de schéma de cohérence territoriale et face à une demande sociale plus affirmée. Les résultats permettent de sensibiliser les élus au contenu de leurs documents d'urbanisme, de mieux comprendre les ressorts de ce type d'action publique, et de mieux cerner le rôle de l'expertise dans la formulation de ces documents d'urbanisme. Au-delà des conclusions issues de l'évaluation, la démarche permet d'identifier les leviers d'action les plus pertinents pour améliorer l'efficacité des documents d'urbanisme dans un objectif de gestion partagée des paysages. 
Fiche informative

\section{Discipline}

Sciences et architecture du paysage

Directeur

Pierre Donadieu, Hervé Davodeau

\section{Université}

AgroParis Tech - Ecole doctorale ABIES

Ecole Nationale Supérieure du Paysage de Versailles-Marseilles

Membres du jury de thèse, soutenue le 09/11/2012

- M. Hervé Davodeau, Maître de conférences, INHP/AgroCampus-Ouest

- M. Pierre Donadieu, Professeur, UPR MA LAREP/ENSP Versailles

- Mme Sophie Le Floch, Chargée de recherche HDR, CEMAGREF

- M. Yves Luginbuhl, Directeur de recherche, UMR LADYSS, CNRS

- M. Patrick Moquay, Directeur de recherche, CEMAGREF

- M. Michel Périgord, Professeur, ICOTEM, Université de Poitiers

\section{Situation professionnelle actuelle}

Chargé de recherche 'paysage et aménagement' au Centre d'Etudes Techniques de l'Equipement du Sud-Ouest

Courriel de l'auteur mailto:labatdidier@yahoo.fr 